\title{
Highly transparent and impact-resistant PMMA nanocomposites reinforced by cellulose nanofibers of pineapple leaves modified by eco-friendly methods
}

\author{
Y. F. Shih ${ }^{1}$, M. Y. Chou ${ }^{1}$, H. Y. Lian $^{2}$, L. R. Hsu 3 , S. M. Chen-Wei ${ }^{4}$ \\ ${ }^{1}$ Department of Applied Chemistry, Chaoyang University of Technology, No. 168, Jifeng E. Rd., Wufeng District, \\ 41349 Taichung, Taiwan \\ ${ }^{2}$ Commercialization and Industry Service Center, Industrial Technology Research Institute, Taiwan \\ ${ }^{3}$ Department of Science Education and Application, National Taichung University of Education \\ ${ }^{4}$ Department of Advanced Coating, Division of Applied Chemistry, Material and Chemical Research Laboratories, \\ Industrial Technology Research Institute, Taiwan
}

Received 27 February 2018; accepted in revised form 7 May 2018

\begin{abstract}
In this study, cellulose nanofibers (CNFs) were derived from waste pineapple leaves with the aim of developing a nanocomposite with high impact strength and transparency. First, CNFs were prepared using an acid-base treatment and 2,2,6,6-tetramethylpiperidin-1-oxyl radical (TEMPO)-mediated oxidation, and then, the surface was modified with polymerized methyl methacrylate (MMA) using an eco-friendly method in an aqueous system. The MMA-modified CNF had an increased contact angle from 12.02 to $57.45^{\circ}$, and the surface hydrophobicity improved the interfacial compatibility between the CNF and polymethyl methacrylate (PMMA) matrix. At 1-3 wt $\%$ of the modified CNF, the impact strength of the nanocomposites was significantly improved by $2.7-22.9 \%$. Moreover, this eco-friendly modification allowed CNFs to disperse homogeneously in the nanocomposite for excellent light transmittance. In conclusion, this eco-friendly modification can replace conventional solvent-based modification, allowing modified CNF to effectively reinforce the PMMA nanocomposite.
\end{abstract}

Keywords: nanocomposites, cellulose nanofibers, pineapple leaves, transparent

\section{Introduction}

A plant is composed of cellulose (50-60\%), hemicellulose (20-30\%), lignin (10-20\%), pectin, and wax. As a sustainable and natural polymer, the annual yields of cellulose in the world are 1010 1011 tons. Derived from plant fibers, cellulose nanofibers (CNF) are defined as a fiber composed of 30-40 long chain cellulose polymers. CNFs usually show widths of a few nanometers and lengths of a hundred nanometers or a few micrometers. Due to the abundant hydrogen bonds existing among the cellulose molecules, CNF develops crystalline and amorphous regions in the fiber. CNF has attracted a lot of attention because of its special properties like high aspect ratio, low density, high strength and stiffness, biodegradability and sustainability. So far, it has demonstrated potential in applications as a nanocomposite, transparent substrate, nanofilter, and gas barrier [1-8]. CNFs can be extracted from plant fibers through nanofibrillation by mechanical or chemical methods. In the mechanical method, shear force is employed on the cellulose by machines like the homogenizer, grinder, or microfluidizer, to destroy the intramolecular interaction for nanofibrillation [9-11]. To improve the nanofibrillation efficiency, the chemical method is introduced before mechanical treatment. 
The conventional chemical method can be executed by alkali, enzyme, or oxidation [12-14]. In a previous study, Isogai group employed 2,2,6,6-tetramethylpiperidine (TEMPO) to efficiently assist oxidation on a cellulose surface to catalyze the hydroxyl groups for carboxyl groups $[15,16]$. In the following mechanical treatment, the carboxylate anions $\left(\mathrm{COO}^{-}\right)$ diminished the hydrogen bonds and enhanced the nanofibrillation efficiency [17].

Compared to traditional composites, CNF-containing nanocomposites have superior reinforcement in mechanical properties, thermal resistance, barrier function, and transparency [1, 2, 4, 18]. Poly(methyl methacrylate) (PMMA) is one of the most important industrial plastics and is also known as acrylic glass due to its high transparency, light weight, and shatter resistance. In addition to a general grade, PMMA is classified as thermal resistant, highly optical, and impact resistant for high specifications. CNF is regarded as an ideal reinforcement material, and it also appears highly transparent in nanocomposites. However, the abundant hydroxyl groups on the cellulose surface make CNF highly polar and difficult to be dispersed in the PMMA matrix. To improve nanocellulose dispersion, the Wu group used DMF solvent to dissolve the PMMA pellet and then, mixed it with cellulose nanocrystals (CNC) [19]. After ultra-sonication treatment, casting, and drying, the CNCPMMA nanocomposite performed with great reinforcement and transparency. However, this solventbased fabrication uses lots of DMF solvent and ultrasonication time and is rarely applied in industrial manufacturing processes. Littunen et al. [7] investigated the grafting reactivities of cerium-initiated copolymerization between acrylic monomers and
CNF in aqueous condition. Subsequently, the obtained PMMA grafted CNF was mixed with PMMA by solution blending. The results reveal that the tensile strength and elongation at break of the PMMA grafted CNF (concentration: $0.5,1.0$ and $5.0 \mathrm{wt} \%$, respectively)/PMMA composites were all lower than those of pristine PMMA [8]. Qin et al. [20] modified the rice straw fiber (RSF) by suspension polymerization of butyl acrylate (BA) monomer. The results indicated that poly(butyl acrylate) (PBA) was adsorbed and coated on RSF. Mechanical properties showed that the tensile strength of PBA modified RSF/PLA composites increased as compared with unmodified ones. In this study, we utilized the suspension polymerization to graft PMMA on the surface of the CNF, due to this method can react directly with the TEMPO method-obtained CNF in aqueous solution. The obtained CNF with a layer of PMMA can improve its compatibility with PMMA matrix. Moreover, as compared with BA (permissible exposure limit-time weighted average (PEL-TWA): $10 \mathrm{ppm}$ ), MMA (PEL-TWA: $100 \mathrm{ppm}$ ) is less toxic, and the obtained polymer layer is expected to be more transparent [21]. As a result, the CNF/PLA nanocomposites exhibited not only a good dispersion of CNFs in the PLA matrices, but an improvement in thermal and mechanical properties. Moreover, an excellent transparent appearance was obtained.

In this study, we extract CNF from pineapple leaves and modify CNF by an eco-friendly method to enhance the interfacial compatibility in the PMMA matrix. In Taiwan, pineapple is one of the most important crops, and the waste from pineapple leaves reaches 60000 tons every year. Pineapple leaf fiber (PLF) contains rich cellulose (up to $81 \%$ ), and extracting

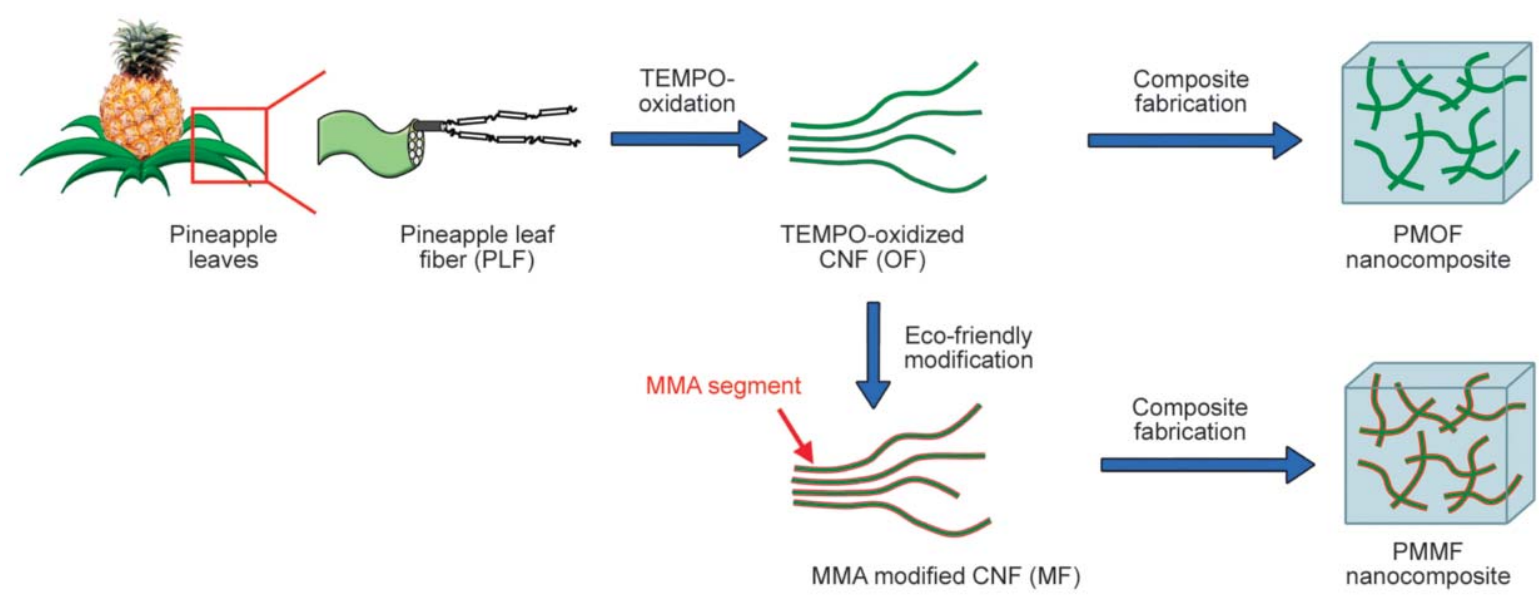

Figure 1. Experimental illustration: preparation of OF, surface modification of MF, and fabrication of nanocomposites. 

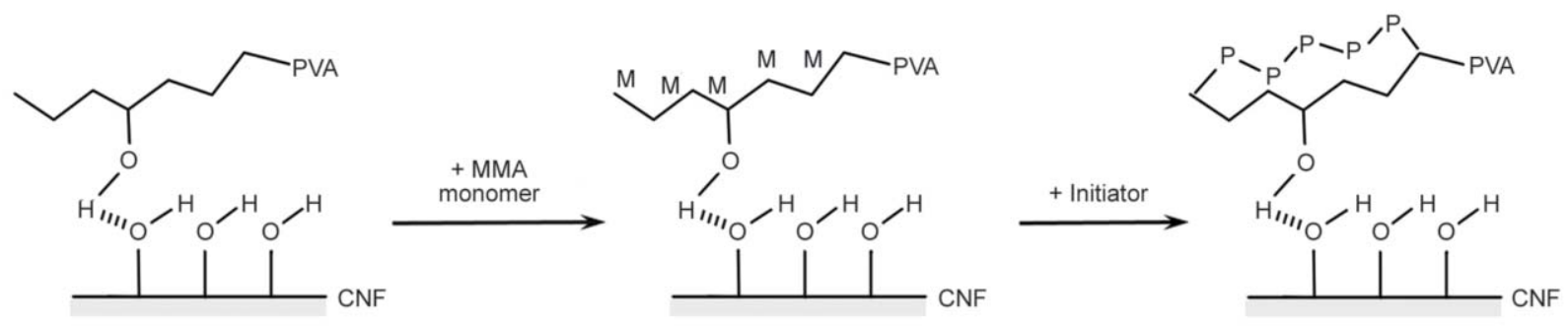

Figure 2. Surface modification of CNF through the suspension polymerization of MMA.

CNF from PLF can reduce agricultural waste. Here, we purify PLF using an acid-base chemical treatment, and extract CNF from PLF by the TEMPO method, as illustrated in Figure 1. To improve the interfacial compatibility between CNF and PMMA, the CNF surface is modified with an MMA (methyl methacrylate) segment in an aqueous system, as illustrated in Figure 2. The aqueous system replaces the organic solvent and takes advantage of the low cost and environmental protection. The modified CNF can be easily blended with a PMMA pellet and shaped by compression molding. The CNF-PMMA nanocomposites are also investigated by analyzing mechanical, thermal, and optical properties.

\section{Experiment}

\subsection{Chemicals}

The PMMA pellets $(\mathrm{CM}-211 \mathrm{G}$ grade, $100000<$ $\left.M_{\mathrm{W}}<300000\right)$ were supplied by Chimei chemical Co., Ltd., Tinan, Taiwan. The raw pineapple leaves were kindly supplied by the Chiayi Agricultural Experiment Station, Taiwan Agricultural Research Institute, Chiayi, Taiwan. Sodium hydroxide $(\mathrm{NaOH})$, hydrochloric acid $(\mathrm{HCl})$, sodium hypochlorite ( $\mathrm{NaClO})$, and acetone were supplied by Echo Chemical Co., Ltd., Miaoli, Taiwan. 2,2,6,6-tetramethylpiperidin-1-oxyl (TEMPO) and polyvinyl alcohol (PVA) were purchased from Sigma-Aldrich Co., Darmstadt, Germany. Methyl methacrylate (MMA), tetrahydrofuran (THF), 2,2'-azobis(2-methylpropionitrile) (AIBN), and sodium chlorite $\left(\mathrm{NaClO}_{2}\right)$ were purchased from Alfa Aesar, Massachusetts, USA, and used without further purification.

\subsection{Preparation of $\mathrm{CNF}$}

\subsubsection{Preparation of PLF}

The raw pineapple leaves were chopped, sun-dried, crushed, and further treated with a $4 \% \mathrm{NaOH}$ solution for $45 \mathrm{~min}$ to remove the lignin, hemicellulose, and impurities. Subsequently, it was neutralized by $1 \% \mathrm{HCl}$ for $5 \mathrm{~min}$, washed with water, and then dried to obtain PLF. The PLF was further bleached in an acetate buffer solution containing $0.3 \% \mathrm{NaClO}_{2}$ at $\mathrm{pH} 4.8,60^{\circ} \mathrm{C}$ for $3 \mathrm{~h}$. At the end, the bleached PLF was purified by water and stored at $4{ }^{\circ} \mathrm{C}$ before further treatment.

\subsubsection{TEMPO-mediated oxidation}

The bleached PLF $(1 \mathrm{~g})$ was suspended in a sodium phosphate buffer $(90 \mathrm{~mL}, \mathrm{pH}$ 6.8) containing TEMPO $(0.016 \mathrm{~g})$ and sodium chlorite $(80 \%, 1.13 \mathrm{~g})$ in an airtight flask. $0.5 \mathrm{~mL}$ of $2 \mathrm{M}$ sodium hypochlorite solution was diluted to $0.1 \mathrm{M}$ with the same buffer, and then, it was added as the oxidation medium. The TEMPO-mediated oxidation was started, and stirring continued at $60^{\circ} \mathrm{C}$ for $3.5 \mathrm{~h}$. After being washed with water, the TEMPO-mediated oxidation and purification were repeated for preparing TEMPO-oxidized CNF (OF). The size of OF synthesized was about $55 \mathrm{~nm}[21]$.

\subsection{Eco-friendly modification of $\mathrm{CNF}$}

An MMA monomer, initiator (AIBN), and dispersant (PVA) were mixed according to the ratio of 1:0.05:0.03 in distilled water, and then, OF was added (The amount of MMA monomer relative to the OF was $18 \mathrm{mmol} \mathrm{MMA} / \mathrm{g} \mathrm{OF})$. The solution was stirred continuously at $75^{\circ} \mathrm{C}$ for $6 \mathrm{~h}$ under a nitrogen atmosphere. At the end, MMA modified fiber (MF) was obtained after filtration, washing with water, then washing with THF to remove the un-grafting part, and being freeze dried [21]. The percentage of weight of PMMA grafted on CNF, $W[\%]$, is calculated from Equation (1) [20]:

$$
W[\%]=\frac{M_{\mathrm{MCNF}}-M_{\mathrm{CNF}}}{M_{\mathrm{CNF}}} \cdot 100
$$

where $M_{\mathrm{CNF}}$ is the original weight of $\mathrm{CNF}, M_{\mathrm{MCNF}}$ is the weight of the CNF after modification.

The percentage of weight of PMMA grafted on CNF calculated from Equation (1) was 14.3\%. 


\subsection{Fabrication of nanocomposites}

The PMMA pellets, MF, and OF were dried in an oven at $55^{\circ} \mathrm{C}$ for 1 day and then, mixed in the ratios shown in Table 1. The CNF (MF or OF) and PMMA pellets were blended at $175^{\circ} \mathrm{C}$ in a counter-rotating internal mixer (Brabender PL2000, Duisburg, Germany) with a rotational speed of $50 \mathrm{rpm}$. After being well blended for 15 min, the PMMF (PMMA reinforced by MF) and PMOF (PMMA reinforced by $\mathrm{OF})$ nanocomposites were granulated. Finally, the nanocomposites were shaped by compression molding at $175^{\circ} \mathrm{C}$.

\subsection{Instrumental analysis}

The FTIR was measured by the PerkinElmer Paragon 500 (PerkinElmer, Inc., Waltham, USA) from 400 to $4000 \mathrm{~cm}^{-1}$, and all samples were taken using the $\mathrm{KBr}$ disk method. Electron spectroscopy for chemical analysis (ESCA) was carried out using the ULVACPHI PHI5000 Versaprobe (ULVAC-PHI Inc., Kanagawa, Japan). The morphology of PLF was observed by a polarizing microscope (POM) (Axio Scope.A1, Carl Zeiss AG, Jena, Germany); the morphology of CNF was observed by a TEM (JEM-2100, JEOL, Ltd., Tokyo, Japan). SEM micrographs were made by a Hitachi S-3000N (Hitachi, Ltd., Tokyo, Japan) after the sample surfaces were sputter-coated with gold to avoid charging. The hydrophilic property of a CNF was measured by contact angle analyzer (FTA125, First Ten Ångstroms, Inc., Virginia, USA) using deionized water as a probing liquid. Thermal behavior was determined using a thermogravimetric analyzer (TGA) (TGA Q50, TA Instruments, Inc., Delaware, USA) and the samples were scanned at a heating rate of $10^{\circ} \mathrm{C} / \mathrm{min}$ in the presence of a nitrogen flow. The tensile test was carried out according to the ASTM D638 test method at a strain rate of

Table 1. Nanocomposites' component.

\begin{tabular}{|l|c|c|c|}
\hline \multirow{2}{*}{ Sample } & \multicolumn{3}{|c|}{ Component } \\
\cline { 2 - 4 } & $\begin{array}{c}\text { PMMA } \\
\text { [wt\%] }\end{array}$ & $\begin{array}{c}\text { MF } \\
{[\mathbf{w t} \%]}\end{array}$ & $\begin{array}{c}\text { OF } \\
{[\mathbf{w t} \%]}\end{array}$ \\
\hline PMMA & 100.0 & - & - \\
\hline PMMF0.5 & 99.5 & 0.5 & - \\
\hline PMMF1 & 99.0 & 1.0 & - \\
\hline PMMF2 & 98.0 & 2.0 & - \\
\hline PMMF3 & 97.0 & 3.0 & - \\
\hline PMOF0.5 & 99.5 & - & 0.5 \\
\hline PMOF1 & 99.0 & - & 1.0 \\
\hline PMOF2 & 98.0 & - & 2.0 \\
\hline PMOF3 & 97.0 & - & 3.0 \\
\hline
\end{tabular}

$50 \mathrm{~mm} / \mathrm{min}$ by an Instron universal tester (HT-9102, Hung Ta Instrument Co., Ltd., Taichung, Taiwan). The impact strength was analyzed according to the ASTM D256 test method using an impact resistance testing machine (GT-70045-MDL, Gotech Testing Machines, Inc., Taichung, Taiwan). The transmittance of a nanocomposite (The thickness of the sample was $1 \mathrm{~mm}$ ) was measured by ultraviolet and visible spectroscopy (SPECORD 200, Analytic Jena AG, Jena, Germany).

\section{Results and discussion}

\subsection{Characterizations of modified CNF}

\subsubsection{FTIR}

To confirm the TEMPO-mediated oxidation and MMA polymerization on the CNF surface, FTIR was used to detect the variation in functional groups, as shown in Figure 3. All fibers display a broad peak at $3400 \sim 3600 \mathrm{~cm}^{-1}$, which is attributed to by the $\mathrm{O}-\mathrm{H}$ stretch of cellulose. In Figure 3 curve a, the absorption peaks at $1735 \mathrm{~cm}^{-1}$ correspond to the $\mathrm{C}=\mathrm{O}$ of lignin [22], and it disappears in Figure 3 curve $b$ after acidbase treatment and TEMPO-mediated oxidation, indicating that most of the lignin and impurities were successfully removed. Figure 3 curve $b$ exhibits the absorption peaks of an alcohol $\mathrm{O}-\mathrm{H}$ stretch, carboxylic acid $\mathrm{O}-\mathrm{H}$ stretch, and carboxylic acid $\mathrm{C}=\mathrm{O}$ stretch at 3450,3325 and $1614 \mathrm{~cm}^{-1}$, respectively.

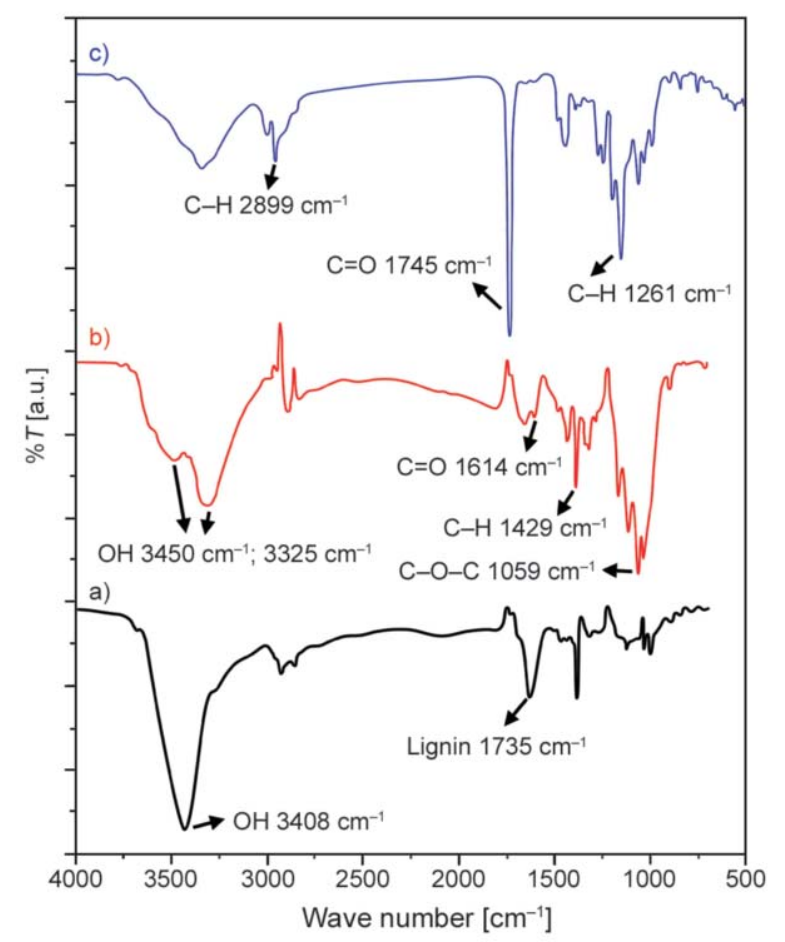

Figure 3. FTIR spectra of (a) pineapple leaf, (b) OF and (c) MF. 
That means that part of the alcohol $\mathrm{O}-\mathrm{H}$ on the CNF surface was successfully catalyzed to carboxylic acid through TEMPO-mediated oxidation. In Figure 3 curve $\mathrm{c}$, the existence of MMA contributes to the significant absorption peaks of $\mathrm{C}-\mathrm{H}$ stretch at $2857 \mathrm{~cm}^{-1}$, $\mathrm{C}=\mathrm{O}$ stretch at $1745 \mathrm{~cm}^{-1}$, and $-\mathrm{C}-\mathrm{H}$ bending at $1261 \mathrm{~cm}^{-1}$. Therefore, it confirms the success of MMA modification on the MF surface.

\subsubsection{ESCA}

The ESCA spectra can be used to quantify the chemical composition of the CNF surface, as shown in

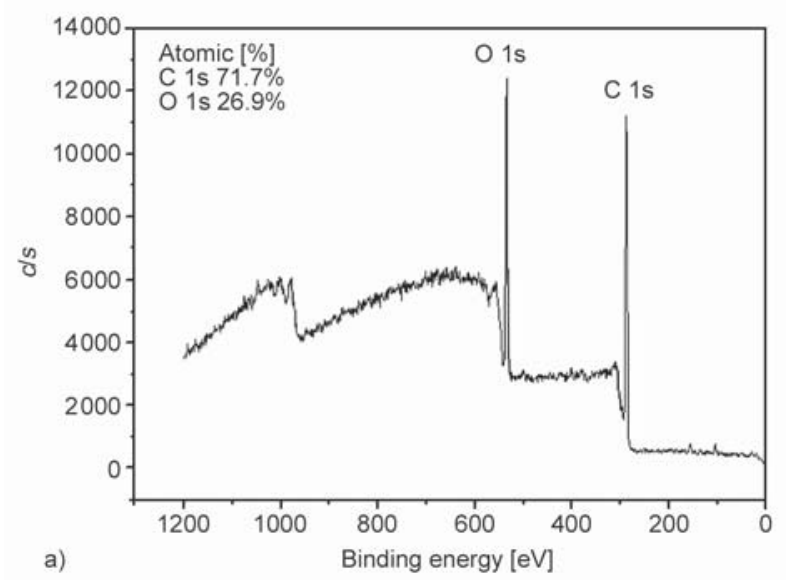

Figure 4. ESCA spectra of (a) OF and (b) MF.
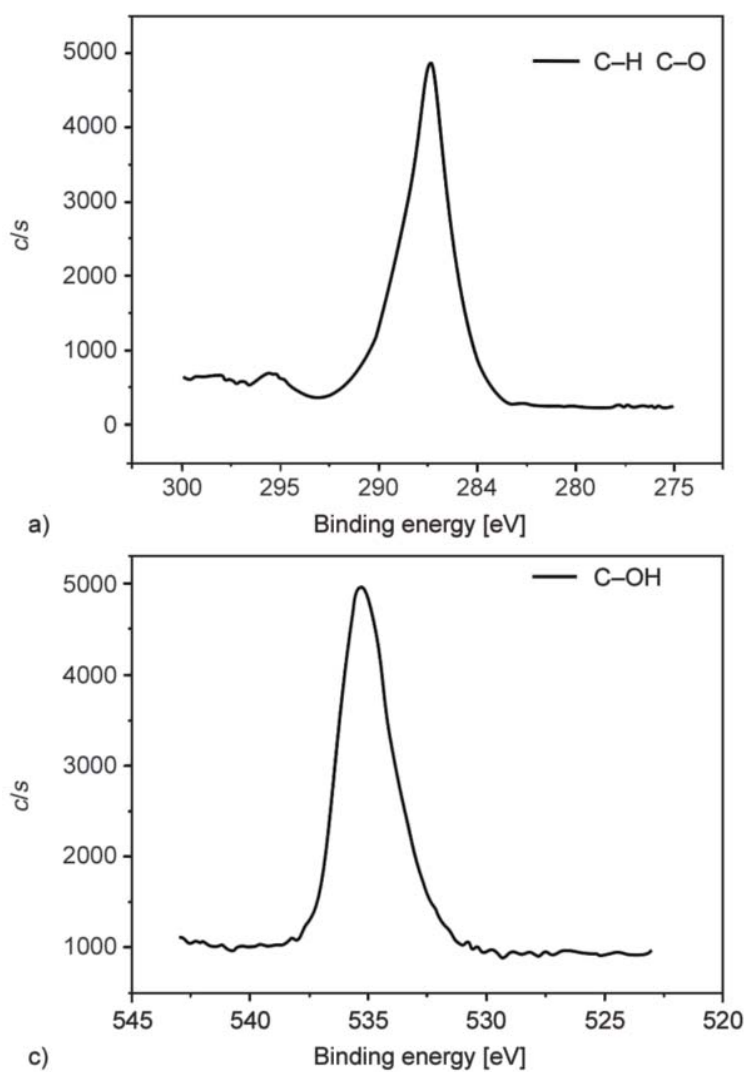

Figure 4. Figure 4a exhibits the electron emission for carbon (C 1s) and oxygen (O 1s) at 535.297 and $287.38 \mathrm{eV}$, respectively. By calculating the atom percent, PLF and OF show oxygen elements of 19.6 and $26.9 \%$, respectively. The oxygen elevation reveals that part of the alcohol $\mathrm{OH}$ groups was successfully oxidized for carboxyl $\mathrm{COOH}$ groups using the TEMPO method. After MMA modification, as shown in Figure 4b, the methyl ester part further increases the oxygen element to $36 \%$.

Different chemical bonding can be investigated by separating the $\mathrm{C} 1 \mathrm{~s}$ and $\mathrm{O} 1 \mathrm{~s}$ peaks, as shown in Figure 5.
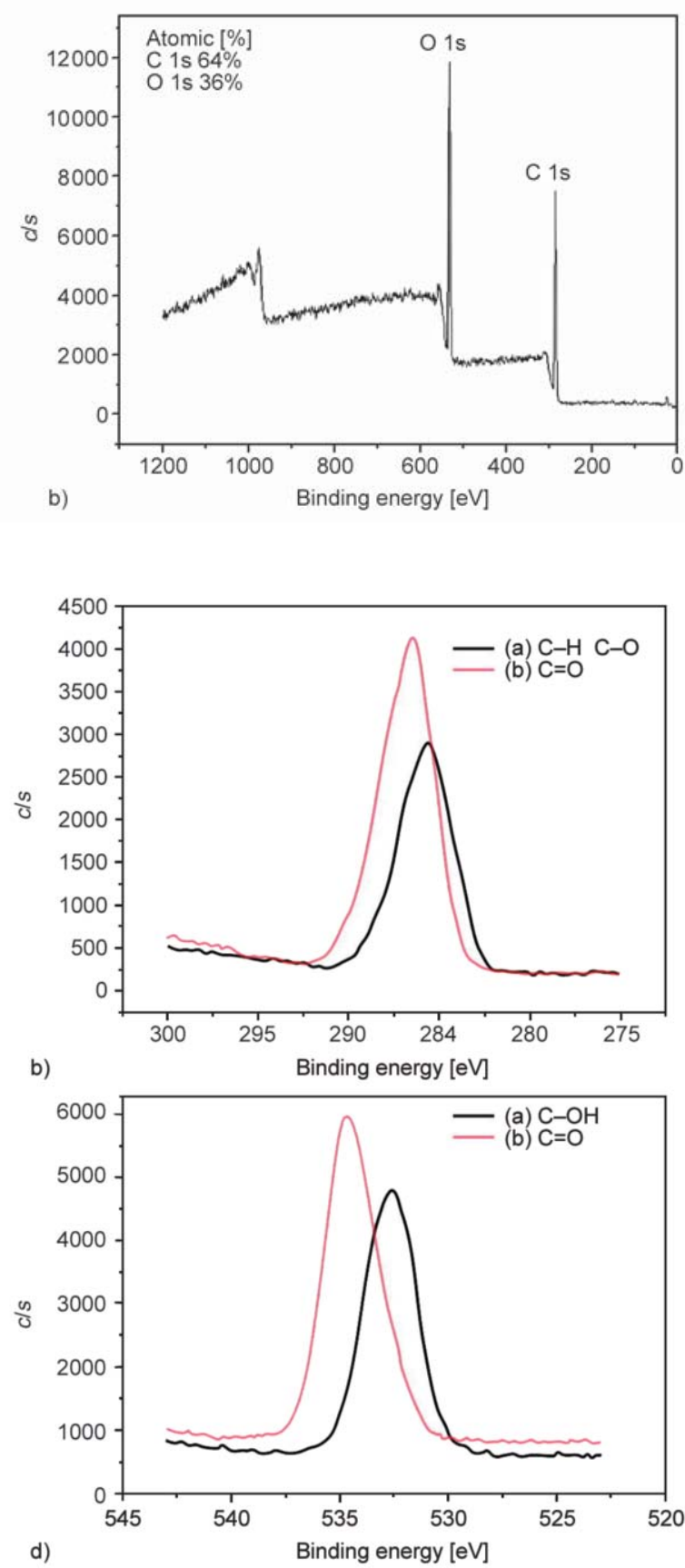

Figure 5. ESCA C 1s spectra of (a) OF and (b) MF, and O 1s spectra of (c) OF and (d) MF. 
In the $\mathrm{C} 1 \mathrm{~s}$ spectrum (Figure $5 \mathrm{a}$ ), the $\mathrm{OF}$ shows $\mathrm{C}-\mathrm{H}$ and $\mathrm{C}-\mathrm{O}$ peaks at 287 and $285 \mathrm{eV}$, respectively. In the $\mathrm{O} 1 \mathrm{~s}$ spectrum of the $\mathrm{OF}$ (Figure $5 \mathrm{c}$ ), the $\mathrm{OH}$ and $\mathrm{C}-\mathrm{OH}$ peaks are at 533 and $535 \mathrm{eV}$, respectively. The chemical bonds in alcohol and alkane above are assigned to the glucose unit of cellulose and exist in both the OF and MF. After MMA modification, the $\mathrm{C} 1 \mathrm{~s}$ and $\mathrm{O} 1 \mathrm{~s}$ spectra of MF are exhibited in Figures $5 \mathrm{~b}$ and $5 \mathrm{~d}$, respectively. The MF displays an extra $\mathrm{C}=\mathrm{O}$ peak at $286 \mathrm{eV}$ in the $\mathrm{C} 1 \mathrm{~s}$ spectrum and $535 \mathrm{eV}$ in the $\mathrm{O} 1 \mathrm{~s}$ spectrum. The $\mathrm{C}=\mathrm{O}$ bond is attributed by the methyl ester of MMA. To summarize, it is confirmed that the MMA segment was successfully grafted onto the MF surface by eco-friendly modification.

\subsubsection{Morphology and surface hydrophilicity}

From the POM observation shown in Figures $6 \mathrm{a}$ and $6 \mathrm{~b}$, cellulose fibers vary in dimension and surface texture after purification by acid-base treatment. In

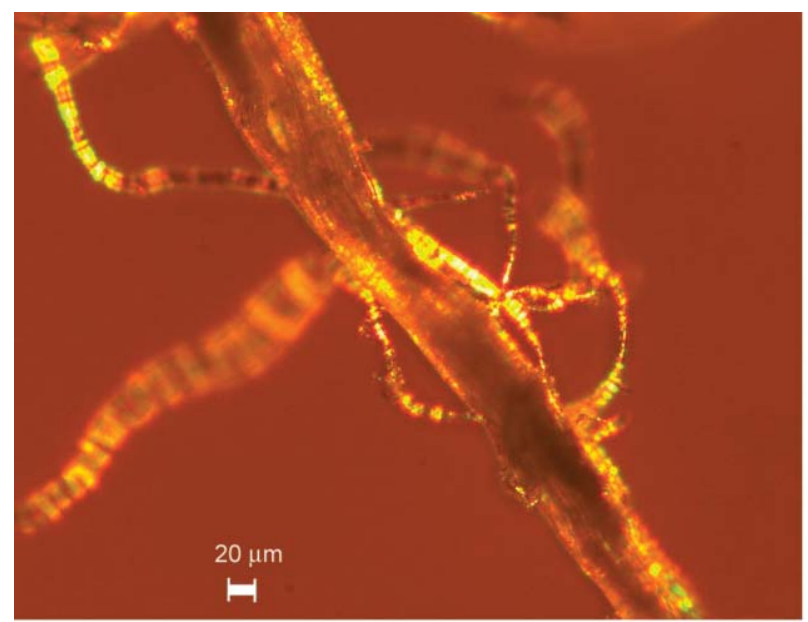

a)
Figure $6 \mathrm{a}$, the raw pineapple leaves are entangled by many fibers and aggregate seriously in a lump. On the surface, the roughness indicates the existence of pectin and lignin. In Figure 6b, the acid-base treatment cuts down the fiber dimension and lessens PF entanglement. Also, the smooth surface reflects that impurities like pectin and lignin were removed by the acid-base treatment. According to previous studies, cellulose can be nanofibrillated for preparing CNF through TEMPO-mediated oxidation assisted with high-speed homogenization $[15,16]$. To improve the compatibility of CNF in a nanocomposite, this study modified the CNF surface with an MMA segment by suspension polymerization. From the TEM image shown in Figure 6c, after nanofibrillation and MMA modification, MF successfully achieves a nano-scale with a width of about $98 \mathrm{~nm}$. In addition, a transparent layer of the MMA segment is found to be coated on the MF surface, illustrating successful MMA modification.

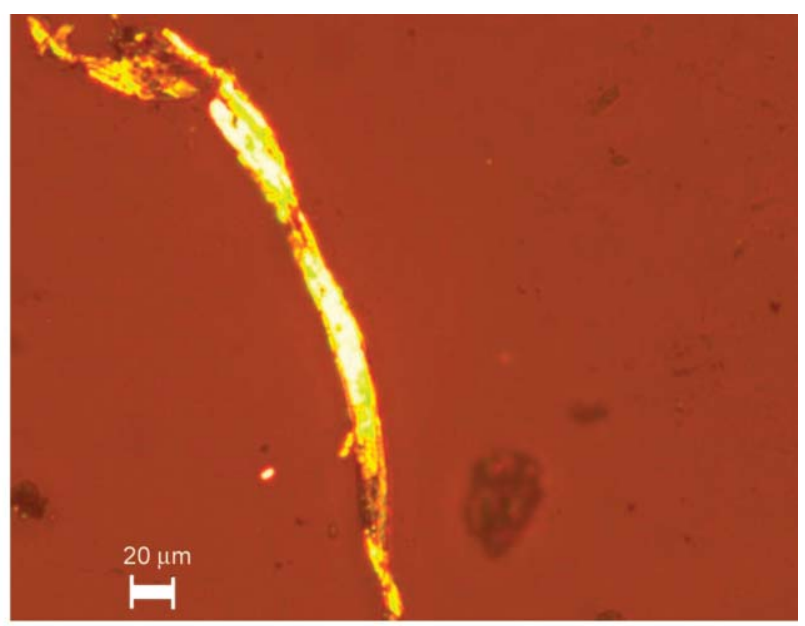

b)

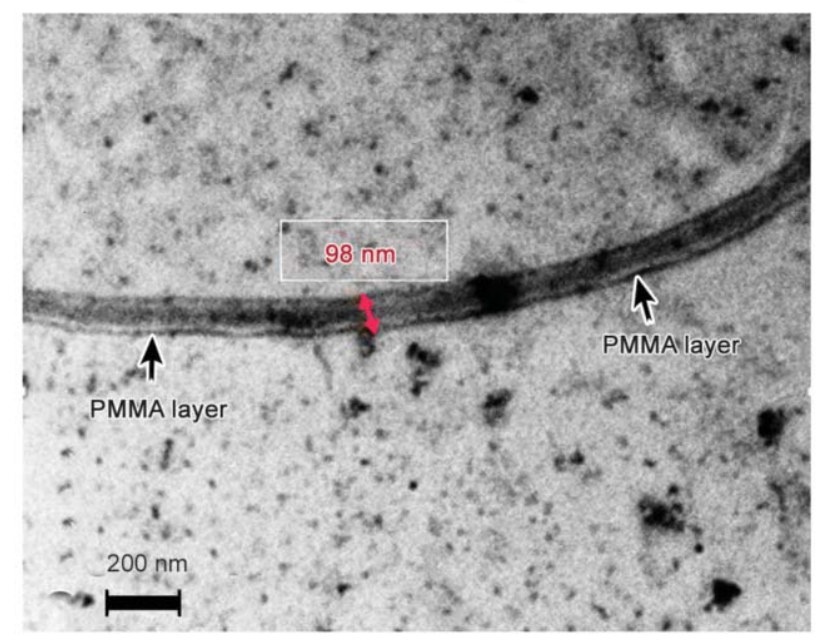

c)

Figure 6. Fiber morphology observed by POM and TEM: POM images of (a) pineapple leaf and (b) PLF, and TEM image of (c) MF. 


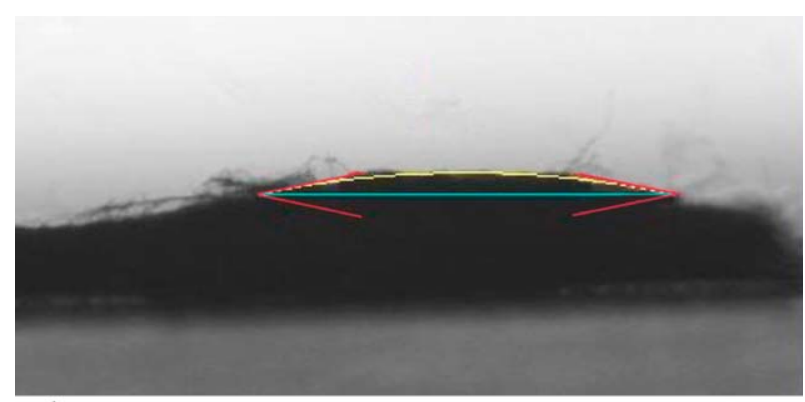

a)

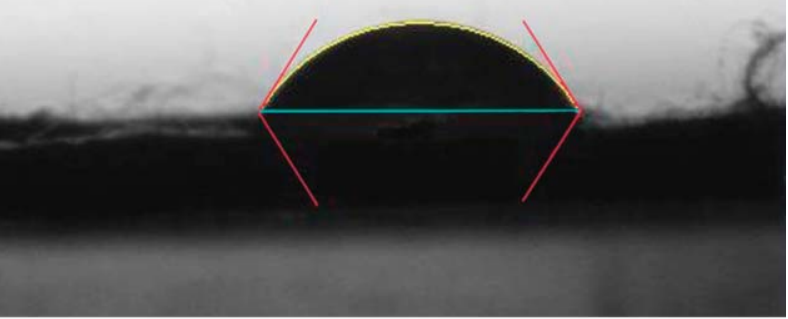

b)

Figure 7. Contact angle of water droplet on (a) OF and (b) MF.

The modified MMA can also be demonstrated by surface hydrophobicity. A cellulose surface is highly polar because of the abundant hydroxyl groups, and that usually results in heterogeneous dispersion of cellulose reinforcement in the polymer matrix. To lower the CNF surface's polarity, MMA was polymerized on the CNF surface. The surface polarity of CNF can be clarified by surface hydrophilicity, and here the surface hydrophilicity is characterized in terms of contact angle, as shown in Figure 7. Figure 7 a displays a low contact angle of $12.02^{\circ}$ on the $\mathrm{OF}$, and that means the OF has an extremely high hydrophilicity and surface polarity. In Figure $7 \mathrm{~b}$, the $\mathrm{PF}$ increases the contact angle to $57.45^{\circ}$, and that explains how the polymerized MMA segment on MF surface can enhance hydrophobicity by long carbon chains. Therefore, the MMA modification lowers the MF's surface polarity and improves MF dispersion in nanocomposites for optimal reinforcement.

\subsubsection{Thermal analysis}

The TGA analysis of fibers is shown in Figure 8. The decomposition temperatures at $5 \%$ weight-loss $\left(T_{\mathrm{d} 5}\right)$ and the maximum degradation temperature $\left(T_{\max }\right)$ and char yield are summarized in Table 2. The PLF (unmodified fiber, UMF) has a $T_{\mathrm{d} 5}$ of $290.61^{\circ} \mathrm{C}$, a $T_{\max }$ of $364.7{ }^{\circ} \mathrm{C}$, and a char yield of $16.223 \%$. The TEMPO-oxidized CNF (OF) has a $T_{\mathrm{d} 5}$ of $261.1^{\circ} \mathrm{C}$, a $T_{\max }$ of $342.9^{\circ} \mathrm{C}$, and a char yield of $7.979 \%$. The

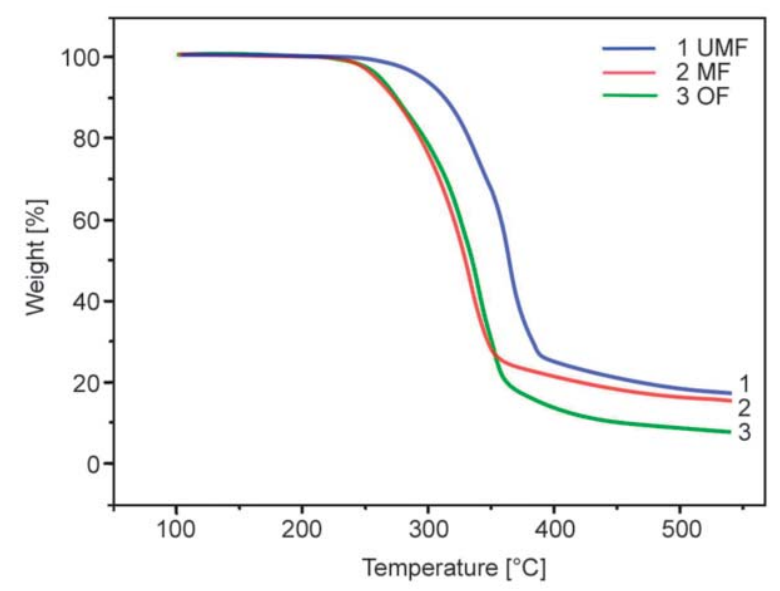

Figure 8. TGA analysis of fibers.

Table 2. TGA properties of fibers and composites.

\begin{tabular}{|l|c|c|c|}
\hline \multicolumn{1}{|c|}{ Sample } & $\begin{array}{c}\boldsymbol{T}_{\mathbf{d} 5} \\
{\left[{ }^{\circ} \mathbf{C}\right]}\end{array}$ & $\begin{array}{c}\boldsymbol{T}_{\max } \\
{\left[{ }^{\circ} \mathbf{C}\right]}\end{array}$ & $\begin{array}{c}\text { Char yield at 530 } \\
{\left[{ }^{\circ} \mathbf{C}\right.}\end{array}$ \\
\hline MF & 257.6 & 334.1 & 15.4800 \\
\hline OF & 261.1 & 342.9 & 7.9790 \\
\hline UMF & 290.6 & 364.7 & 16.2230 \\
\hline PMMA & 331.3 & 371.4 & 0.0089 \\
\hline PMMF0.5 & 336.1 & 372.3 & 0.2958 \\
\hline PMMF1 & 337.4 & 373.9 & 0.4238 \\
\hline PMMF2 & 339.8 & 375.2 & 0.8681 \\
\hline PMMF3 & 342.5 & 376.2 & 1.0080 \\
\hline PMOF0.5 & 332.3 & 370.2 & 0.2807 \\
\hline PMOF1 & 337.1 & 373.8 & 0.2958 \\
\hline PMOF2 & 338.3 & 374.2 & 0.6570 \\
\hline PMOF3 & 335.2 & 373.5 & 0.8217 \\
\hline
\end{tabular}

formation of sodium carboxylate groups from the C6 primary hydroxyls on OF leads to a decrease in the thermal degradation point [23]. After MMA modification, the MF decreases the $T_{\mathrm{d} 5}$ and $T_{\max }$ slightly to 257.6 and $334.1^{\circ} \mathrm{C}$, respectively, and dramatically increases the char yield to $15.48 \%$. The PMMA segment possesses a much lower $T_{\mathrm{d} 5}$ and $T_{\max }$ than those of OF [24, 25] and accordingly, reduces the MF's decomposition temperature. During thermal decomposition, the PMMA segment decomposed and carbonized, and the carbon layer protected the inner fiber from further decomposition. As a result, the inner fiber carbonized and elevated the char yield to twice that of OF.

It is shown in Table 2 that the $T_{\mathrm{d} 5}, T_{\max }$ and char yield of PMMF nanocomposites are all higher than those of pristine PMMA and PMOF nanocomposites. This indicates that the addition of MF improves the thermal properties of PMMA more effectively than that of OF. 


\subsection{Mechanical properties of nanocomposites}

\subsubsection{Tensile test}

The tensile test data of nanocomposites containing $0.5 \sim 3 \% \mathrm{CNF}$ is summarized in Table 3. Except PMOF0.5, the modulus of the composites is all higher than that of pristine PMMA, indicating that the rigidity is increased by addition of CNF. Especially, the modulus of the composites is significantly increased when the CNF content is larger than $1 \mathrm{wt} \%$. However, the elongation at break of the composites is all lower than that of pristine PMMA due to the nature of fiber. At the same CNF content, all PMOF nanocomposites show a lower tensile strength, modulus and elongation at break than those of PMMF ones. While nanocomposites withstand external force, the stress is delivered from the matrix to the CNF. During the process, the compatibility and adhesion between the CNF and PMMA matrix decide the reinforcement effect. At a $0.5-3 \%$ OF content, PMOF nanocomposites decrease tensile strengths by -21.3 to $-8.7 \%$. According to the contact angle test, $\mathrm{OF}$ has a high surface hydrophilicity and polarity. Therefore, OF easily creates poor compatibility and defects in PMOF nanocomposites, as confirmed in Figure 9a. In the end, the defects and poor adhesion cause failure in the OF reinforcement and decrease tensile strength.

In PMMF nanocomposites, the tensile strength is improved while the MF additive becomes greater than $1 \%$. According to the contact angle test, MF enhances surface hydrophobicity and lowers polarity. Hence, the MMA segment on the MF surface benefits the interfacial compatibility and adhesion between the MF and PMMA matrix. As verified in Figure 9b, the PMMF nanocomposite displays a dense texture, and the MF is well embedded in the PMMA matrix. However, the hydrogen bonding between the CNF and MMA segment, as illustrated in Figure 2, barely

Table 3. Nanocomposites' mechanical properties.

\begin{tabular}{|c|c|c|c|c|c|c|}
\hline \multirow[t]{2}{*}{ Sample } & \multicolumn{2}{|c|}{ Tensile strength } & \multirow{2}{*}{$\begin{array}{c}\text { Elongation } \\
\text { at break } \\
{[\%]}\end{array}$} & \multirow{2}{*}{$\begin{array}{c}\text { Modulus } \\
\text { [GPa] }\end{array}$} & \multicolumn{2}{|c|}{ Impact strength } \\
\hline & $\begin{array}{c}\text { Tensile strength } \\
{[\mathrm{MPa}]}\end{array}$ & $\begin{array}{c}\text { Improvement } \\
{[\%]}\end{array}$ & & & $\begin{array}{c}\text { Impact strength } \\
{[\mathbf{J} / \mathbf{m}]}\end{array}$ & $\begin{array}{c}\text { Improvement } \\
{[\%]}\end{array}$ \\
\hline PMMA & $43.5 \pm 0.3$ & - & $12.95 \pm 0.20$ & $2.35 \pm 0.02$ & $14.8 \pm 0.2$ & - \\
\hline PMMF0.5 & $35.3 \pm 0.5$ & -18.8 & $9.72 \pm 0.32$ & $2.58 \pm 0.10$ & $15.2 \pm 0.7$ & 2.7 \\
\hline PMMF1 & $44.2 \pm 1.2$ & 1.6 & $9.52 \pm 0.30$ & $3.02 \pm 0.08$ & $18.2 \pm 1.3$ & 22.9 \\
\hline PMMF2 & $44.1 \pm 0.9$ & 1.4 & $9.36 \pm 0.36$ & $3.31 \pm 0.10$ & $16.5 \pm 0.4$ & 11.5 \\
\hline PMMF3 & $43.9 \pm 2.2$ & 0.9 & $9.20 \pm 0.42$ & $3.58 \pm 0.12$ & $15.4 \pm 2.3$ & 4.1 \\
\hline PMOF0.5 & $34.2 \pm 1.4$ & -21.3 & $8.96 \pm 0.45$ & $2.23 \pm 0.11$ & $16.9 \pm 1.8$ & 14.2 \\
\hline PMOF1 & $39.7 \pm 0.6$ & -8.7 & $8.97 \pm 0.33$ & $2.78 \pm 0.07$ & $15.3 \pm 0.7$ & 3.4 \\
\hline PMOF2 & $38.7 \pm 1.6$ & -11.0 & $9.05 \pm 0.47$ & $3.03 \pm 0.13$ & $14.2 \pm 0.4$ & -4.1 \\
\hline PMOF3 & $36.1 \pm 2.1$ & -17.0 & $9.01 \pm 0.62$ & $3.12 \pm 0.18$ & $13.7 \pm 1.2$ & -7.4 \\
\hline
\end{tabular}

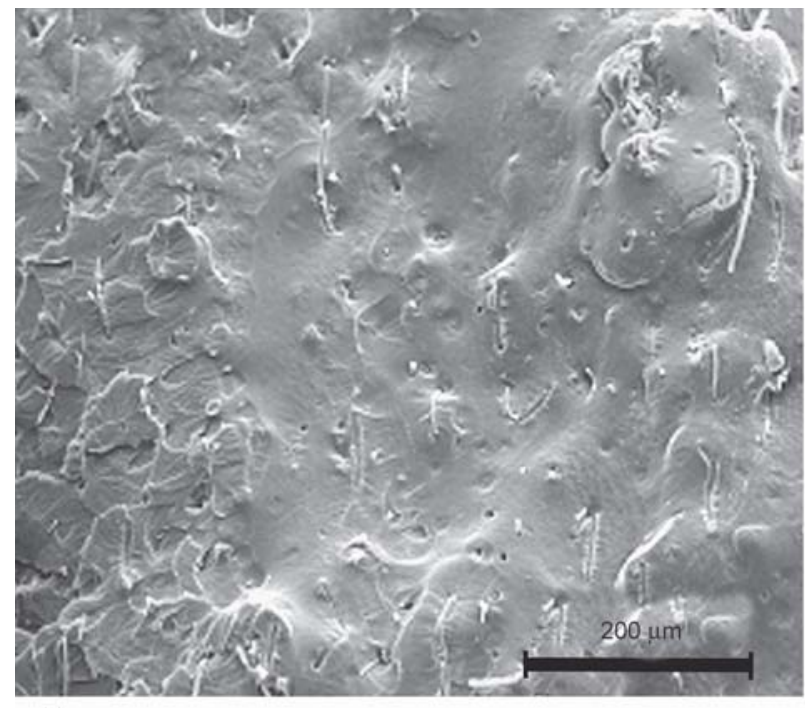

a)

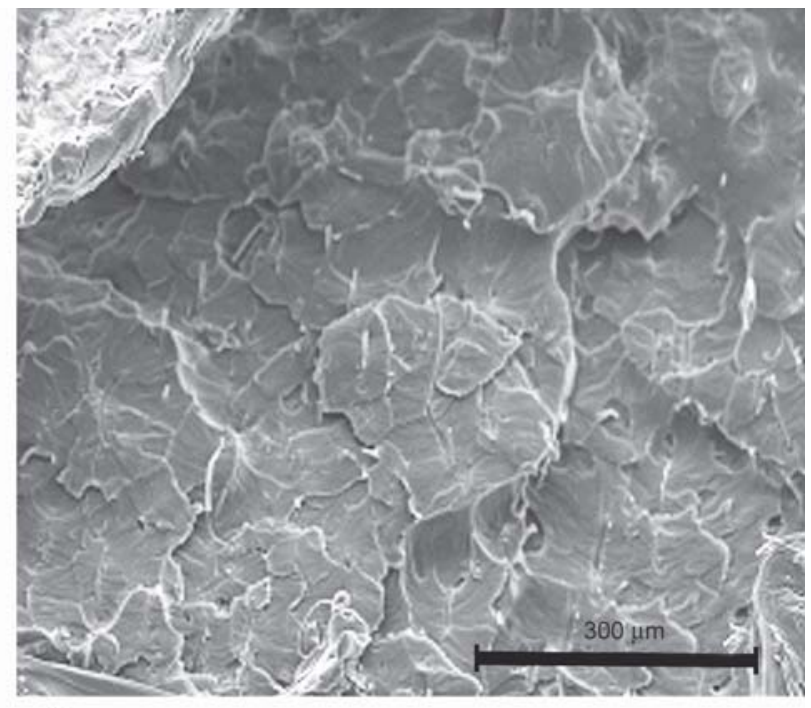

b)

Figure 9. SEM images of nanocomposites' fractured surface after mechanical tests: (a) PMOF1 and (b) PMMF1 after impact test. 
sustains a high tensile stress. In the end, PMMF nanocomposites slightly improve the tensile strength by only a $1.6 \%$ increment at a $1.0 \%$ MF content. Although PMMF1 showed only a slight improvement of only $1.6 \%$, but as compared with other literature [8], it appeared better compatibility with PMMA.

\subsubsection{Impact strength}

As summarized in Table 3, at a $0.5-3 \%$ CNF content, PMOF and PMMF nanocomposites enhance the impact strength by $-7.4 \sim 14.2$ and $2.7 \sim 22.9 \%$, respectively. Under the impact test, CNF can tolerate a high compression strain due to the hollow tube structure of cellulose [26]. In PMOF nanocomposites, PMOF0.5 performs the best impact reinforcement at an increment of $14.2 \%$. At a low OF content, the defects and $\mathrm{CNF}$ aggregations are limited, and therefore, it results in better impact resistance than those with high OF content. For PMMF nanocomposites (exclude the PMMF0.5), all impact strengths are more significantly improved than that of PMOF nanocomposites at the same CNF content. Zhang et al. [27] investigated the nanofibrillated cellulose (NFC) reinforced poly( $\varepsilon$-caprolactone) (PCL)/epoxy composites. The results also showed that the impact strength was improved significantly by adding a small amount of unmodified NFC $(0.3 \mathrm{wt} \%)$. It is largely due to the hollow structure of the fiber providing the anti-vibration effect. On the other hand, the effect of hollow structure of fiber may somewhat be depressed by MMAmodification in this study. So the impact strength of PMMF composites is lower than that of PMOF ones at lower CNF content. However, the better interfacial compatibility becomes the dominant factor to increase the reinforcing effect for MMA-modification $\mathrm{CNF}$ at higher fiber content in the composites. So the impact strength of PMMF composites become larger than that of PMOF ones. It is inferred that MMA surface modification plays an important role for optimizing reinforcement to absorb more impact energy. In PMMF nanocomposites, PMMF1 performs the best enhancement at $22.9 \%$, much higher than PMOF1 (3.4\% increment).

\subsection{Nanocomposites' texture and transparency}

After an impact test, the nanocomposites' fracture surfaces were observed by SEM, as shown in Figure 9. In Figure 9a, PMOF1 contains many micropores, and OF fibers withdraw from the PMMA matrix. The micropores yield defects during impact tests and easily expand to generate cracks, lowering mechanical properties. The withdrawn OF fibers demonstrate poor interfacial compatibility and adhesion between the OF and PMMA matrix. As a result, OF barely absorbs tensile and impact energy and fails to reinforce the nanocomposite. In Figure 9b, PMMF1 displays a condensed texture and a well-embedded MF at the fracture surface, implying that the MMA segment serves as a compatibilizer between the MF and PMMA matrix. During the impact test, the homogenously dispersed MF can hinder crack initiation resulting in a crack deflection mechanism followed by late rupture of the nanocomposite.

Nanocomposites' transmittance and appearance can be observed in Figure 10, respectively. In Figure 10a, PMMF nanocomposites show a high transmittance, close to that of pristine PMMA, and PMMF0.5 performs the highest transmittance among all nanocomposites. In Figure 10b, PMMA, PMMF0.5, and PMMF1 seem highly transparent to the naked eye. The surface modification also reflects a difference
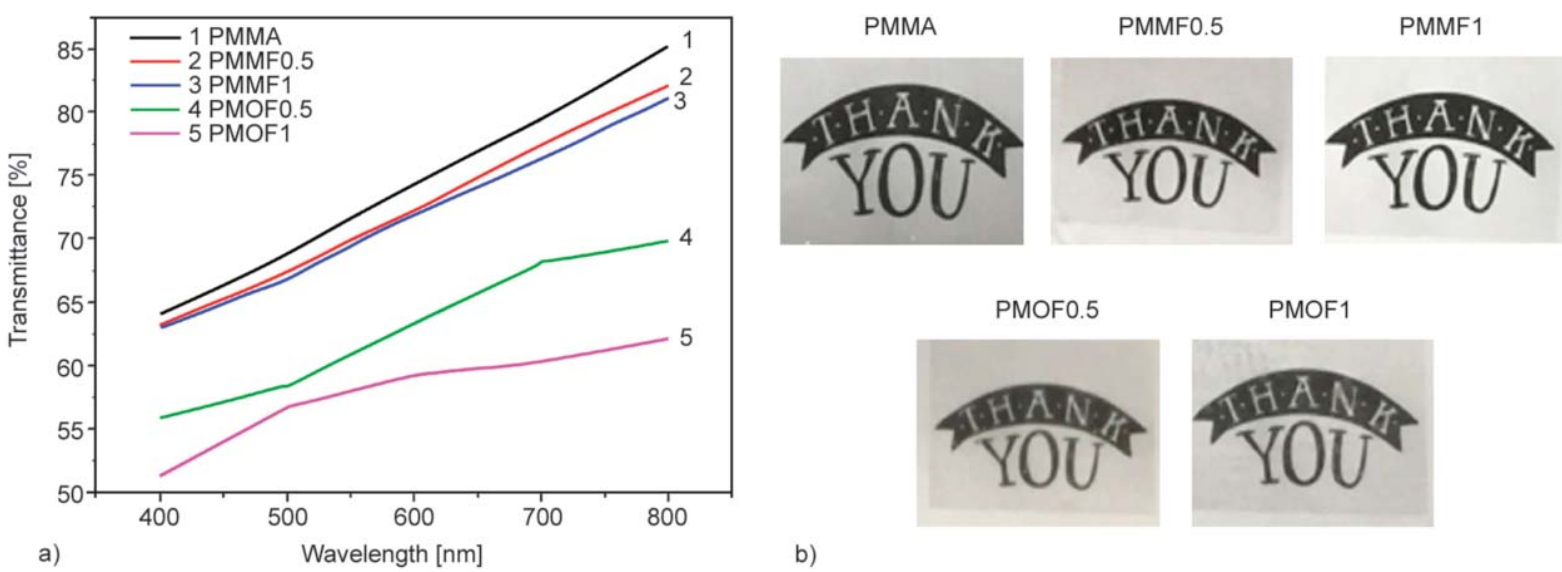

Figure 10. (a) Transmittance and (b) visual examination (THANK YOU logo) of pristine PMMA and nanocomposites. 
in nanocomposite transparency. At the same CNF content, PMMF nanocomposites are more transparent than PMOF nanocomposites. Due to surface hydrophilicity, OF easily aggregates in the PMMA matrix resulting in PMOF nanocomposites with a low transparency and blurred appearance. On the other hand, MF benefits include outstanding CNF dispersity in nanocomposites and maintaining PMMF nanocomposites with a high transparency and clear appearance.

\section{Conclusions}

This study successfully extracted CNF from pineapple leaves through acid-base treatment and TEMPOmediated oxidation. After surface modification with a layer from a segment of MMA created by suspension polymerization, the modified CNF (MF) enhanced hydrophobicity and elevated char yields to $15.48 \%$. The surface modification improved the interfacial compatibility and adhesion between the MF and PMMA matrix, resulting in nanocomposites with higher thermal resistance, mechanical properties, and transparency than those of unmodified CNF. At $1 \mathrm{wt} \%$ MF, PMMF nanocomposite's impact strengths were improved by $22.9 \%$, and excellent light transmittance was kept close to pristine PMMA. Therefore, this research successfully employed an eco-friendly modification for improving the CNF compatibility in PMMA matrix, and enhanced nanocomposite in mechanical, thermal, and optical properties.

\section{Acknowledgements}

This study is financially supported by the Ministry of Science and Technology of Taiwan (MOST 104-2221-E-324-024-). The Chiayi Agricultural Experiment Station of the Taiwan Agricultural Research Institute is appreciated for the kindly supply of pineapple leaves used in this study.

\section{References}

[1] Okahisa Y., Yoshida A., Miyaguchi S., Yano H.: Optically transparent wood-cellulose nanocomposite as a base substrate for flexible organic light-emitting diode displays. Composites Science and Technology, 69, 19581961 (2009).

https://doi.org/10.1016/j.compscitech.2009.04.017

[2] Siró I., Plackett D.: Microfibrillated cellulose and new nanocomposite materials: A review. Cellulose, 17, 459494 (2010).

https://doi.org/10.1007/s10570-010-9405-y
[3] Metreveli G., Wågberg L., Emmoth E., Belák S., Strømme M., Mihranyan A.: A size-exclusion nanocellulose filter paper for virus removal. Advanced Healthcare Materials, 3, 1546-1550 (2014). https://doi.org/10.1002/adhm.201300641

[4] Fukuzumi H., Saito T., Iwata T., Kumamoto Y., Isogai A.: Transparent and high gas barrier films of cellulose nanofibers prepared by TEMPO-mediated oxidation. Biomacromolecules, 10, 162-165 (2009).

https://doi.org/10.1021/bm801065u

[5] Pickering K. L., Efendy M. G. A., Le T. M.: A review of recent developments in natural fibre composites and their mechanical performance. Composites Part A: Applied Science and Manufacturing, 83, 98-112 (2016). https://doi.org/10.1016/j.compositesa.2015.08.038

[6] Littunen K., Kilpeläinen P., Junka K., Sipponen M., Master E. R., Seppälä J.: Effect of xylan structure on reactivity in graft copolymerization and subsequent binding to cellulose. Biomacromolecules, 16, 1102-1111 (2015).

https://doi.org/10.1021/bm501732b

[7] Littunen K., Hippi U., Johansson L-S., Österberg M., Tammelin T., Laine J., Seppälä J.: Free radical graft copolymerization of nanofibrillated cellulose with acrylic monomers. Carbohydrate Polymers, 84, 1039-1047 (2011).

https://doi.org/10.1016/j.carbpol.2010.12.064

[8] Littunen K., Hippi U., Saarinen T., Seppälä J.: Network formation of nanofibrillated cellulose in solution blended poly(methyl methacrylate) composites. Carbohydrate Polymers, 91, 183-190 (2013). https://doi.org/10.1016/j.carbpol.2012.08.032

[9] Lavoine N., Tabary N., Desloges I., Martel B., Bras J.: Controlled release of chlorhexidine digluconate using $\beta$-cyclodextrin and microfibrillated cellulose. Colloids and Surfaces B: Biointerfaces, 121, 196-205 (2014). https://doi.org/10.1016/j.colsurfb.2014.06.021

[10] Iwamoto S., Nakagaito A. N., Yano H.: Nano-fibrillation of pulp fibers for the processing of transparent nanocomposites. Applied Physics A, 89, 461-466 (2007). https://doi.org/10.1007/s00339-007-4175-6

[11] Lavoine N., Desloges I., Dufresne A., Bras J.: Microfibrillated cellulose - Its barrier properties and applications in cellulosic materials: A review. Carbohydrate Polymers, 90, 735-764 (2012). https://doi.org/10.1016/j.carbpol.2012.05.026

[12] Abe K., Iwamoto S., Yano H.: Obtaining cellulose nanofibers with a uniform width of $15 \mathrm{~nm}$ from wood. Biomacromolecules, 8, 3276-3278 (2007). https://doi.org/10.1021/bm700624p

[13] Tibolla H., Pelissari F. M., Rodrigues M. I., Menegalli F. C.: Cellulose nanofibers produced from banana peel by enzymatic treatment: Study of process conditions. Industrial Crops and Products, 95, 664-674 (2017). https://doi.org/10.1016/j.indcrop.2016.11.035 
[14] Isogai A., Saito T., Fukuzumi H.: TEMPO-oxidized cellulose nanofibers. Nanoscale, 3, 71-85 (2011). https://doi.org/10.1039/C0NR00583E

[15] Saito T., Hirota M., Tamura N., Kimura S., Fukuzumi H., Heux L., Isogai A.: Individualization of nano-sized plant cellulose fibrils by direct surface carboxylation using TEMPO catalyst under neutral conditions. Biomacromolecules, 10, 1992-1996 (2009).

https://doi.org/10.1021/bm900414t

[16] Saito T., Nishiyama Y., Putaux J-L., Vignon M., Isogai A.: Homogeneous suspensions of individualized microfibrils from TEMPO-catalyzed oxidation of native cellulose. Biomacromolecules, 7, 1687-1691 (2006). https://doi.org/10.1021/bm060154s

[17] Oksman K., Aitomäki Y., Mathew A. P., Siqueira G., Zhou Q., Butylina S., Tanpichai S., Zhou X., Hooshmand S.: Review of the recent developments in cellulose nanocomposite processing. Composites Part A: Applied Science and Manufacturing, 83, 2-18 (2016). https://doi.org/10.1016/j.compositesa.2015.10.041

[18] Saba N., Mohammad F., Pervaiz M., Jawaid M., Alothman O. Y., Sain M.: Mechanical, morphological and structural properties of cellulose nanofibers reinforced epoxy composites. International Journal of Biological Macromolecules, 97, 190-200 (2017). https://doi.org/10.1016/j.ijbiomac.2017.01.029

[19] Liu H., Liu D., Yao F., Wu Q.: Fabrication and properties of transparent polymethylmethacrylate/cellulose nanocrystals composites. Bioresource Technology, 101, 5685-5692 (2010). https://doi.org/10.1016/j.biortech.2010.02.045

[20] Qin L., Qiu J., Liu M., Ding S., Shao L., Lü S., Zhang G., Zhao Y., Fu X.: Mechanical and thermal properties of poly(lactic acid) composites with rice straw fiber modified by poly(butyl acrylate). Chemical Engineering Journal, 166, 772-778 (2011).

https://doi.org/10.1016/j.cej.2010.11.039
[21] Shih Y-F., Ciou M-Y., Chang W-C., Lian H-Y., Chen CM.: Completely biodegradable composites reinforced by the cellulose nanofibers of pineapple leaves modified by eco-friendly methods. Journal of Polymer Research, 24, 209/1-209/12 (2017).

https://doi.org/10.1007/s10965-017-1367-4

[22] Magniez K., Voda A. S., Kafi A. A., Fichini A., Guo Q., Fox B. L.: Overcoming interfacial affinity issues in natural fiber reinforced polylactide biocomposites by surface adsorption of amphiphilic block copolymers. ACS Applied Materials and Interfaces, 5, 276-283 (2013). https://doi.org/10.1021/am302013p

[23] Fukuzumi H., Saito T., Okita Y., Isogai A.: Thermal stabilization of TEMPO-oxidized cellulose. Polymer Degradation and Stability, 95, 1502-1508 (2010) https://doi.org/10.1016/j.polymdegradstab.2010.06.015

[24] Kashiwagi T., Inaba A., Brown J. E.: Differences in PMMA degradation characteristics and their effects on its fire properties. Fire Safety Science-Proceedings of the First International Symposium, 1, 483-493 (1986). https://doi.org/10.3801/IAFSS.FSS.1-483

[25] Costache M. C., Wang D., Heidecker M. J., Manias E., Wilkie C. A.: The thermal degradation of poly(methyl methacrylate) nanocomposites with montmorillonite, layered double hydroxides and carbon nanotubes. Polymers for Advanced Technologies, 17, 272-280 (2006). https://doi.org/10.1002/pat.697

[26] Kalia S., Dufresne A., Cherian B. M., Kaith B. S., Avérous L., Njuguna J., Nassiopoulos E.: Cellulose-based bio- and nanocomposites: A review. International Journal of Polymer Science, 2011, 837875/1-837875/35 (2011). https://doi.org/10.1155/2011/837875

[27] Zhang Y., Song P., Liu H., Li Q., Fu S.: Morphology, healing and mechanical performance of nanofibrillated cellulose reinforced poly( $\varepsilon$-caprolactone)/epoxy composites. Composites Science and Technology, 125, 6270 (2016).

http://doi.org/10.1016/j.compscitech.2016.01.008 